\title{
Le choix des étudiants candidats aux études de médecine : enjeux sociaux et pédagogiques d'une décision académique
}

'étude de Chantal Kohler et coll. ${ }^{1}$, qui est présentée dans ce numéro, sintéresse au profil académi que des étudiants de dix promotions consécutives de la Faculté de médecine de $N$ ancy, ayant passéle concoursorganiséà la fin dela première année du premier cycle. Elle apporte des donnéesfactuellesqui confirment un certain nombre de notions jusque là implicitement admises ou simplement soupçonnées Ses résul tatsméritent incontes tablement attention dela part detous les enseignants des facultés de médecine françaises et, plus largement, de celle des responsables politiques de ce pays. Au-delà de la particularité française, ils incitent en outre l'ensemble de la communauté médicale internationale à réfléchir aux enjeux de la sélection des étudiants en médecine

Il est important d'avoir à l'esprit que, fondamentalement, cette sélection es d'abord une pratique sociale avant d'êreun problème strictement pédagogique. Diverses tensions sexpriment régulièrement autour de cette pratique, notamment entre une certaine force conservatrice qui tend à perpétuer un statu quo professionnel corporatiste et l'exigence, de plus en plus nettement formulée, d'une plus grande démocratie. II es devenu aujourd'hui commun de consater -et éventuellement de dénoncer sur un mode nostalgique la perte de respectabilité dont seraient victimes les médecins. Force est cependant d'admettre que la profession médicale, de par le monde, reste socialement fortement valorisée Les médecins revendiquent -et bénéfi dent de fait- de la confiance du public, d'un statut social enviable et, d'une manière générale, d'un niveau de revenus très supérieur à celui de la population générale. Ainsi, pour un individu, les conséquences personnelles inhérentes au fait d'être autorisé à entamer ou à poursuivredesétudes médicalesvont bien au-delà de la simple perspective de devenir un jour « docteur ».
Ellescomprennent également la promesse de recevoir, à terme, tout un ensemble d'attributs sociaux qui confèrent une indiscutable capacité d'influence dans des domaines variés, professionnels ou non ; en font partie, par exemple, l'introduction dans diverses sociétés scientifiques mais aussi dans divers cercles intellectues, politiques ou sociaux, l'accès aux medias, à des fondations privées ou à des agences gouvernementales ${ }^{2}$.

Ceci explique la variété des dispositifs adoptés pour affronter le problème de l'écart entre le grand nombre de candidats à la profession médicale et le nombre infiniment plus restreint de professionnels diplômés qu'une société estime nécessaire de recruter, à un moment donné, pour résoudre avec efficacitéet efficience les problèmes de santé de sa population. Ces di spositifs sont en effet toujours très étroitement contingents des valeurs véhiculées par les contextes sociaux, politiques, économiques et culturels. Dans la Chine ancienne ains que dans la G rèce et la Rome antiques", cette sélection avait généralement un caractère héréditaire et privilégiait le plus souvent ledroit d'aînesse D ansl'E urope du M oyen-âge sont apparues des règles rigides faisant référence à une sorte de code de conduite morale. $D$ ans les pays occidentaux, la période actuelle et globalement caractérisée par un contexte hautement compétitif ; les dédions sy appuient, selon des combinaisons variables sur tout ou partie des résultats scolaires obtenus pendant les études secondaires, sur des mesures psychométriques évaluant diverses habiletés intellectuelles et sur l'analyse de certains traits individues se référant au caractère et aux qualités personnelles desimpétrants ${ }^{5}$. M ais, même aujourd'hui, les exigences de la notion d'équité sont diversement appréd ées Au sein des nationsqui érigent en valeur sociale prééminente la référence communautarise, l'objectif de ne désavantager aucune communauté 
ethnique fait, par exemple, l'objet d'une préoccupation scrupuleuse ${ }^{6}$; au nom du principe de discrimination positive (affirmative action), il peut même conduire à instaurer des quotas pour favoriser l'accès des études médicales à des étudiants issus de groupes sociaux ou ethniques historiquement défavorisés'. En France, au regard de l'idéal universaliste et républicain prodamé, de tellesmesures serai ent tout simplement inacceptables

Le processus de sélection des étudiants en médecine vise en fait, implicitement ou explicitement, deux types de buts. Les uns, à court terme, concernent la progression de l'éudiant dans le cursus des études médicales Les autres, à long terme, concernent les conséquences qu'auront les caractéristiques des éudiants promus à l'égard des orientations futures du système de santé, de la recherche bio-clinique $\notin$ médico-psycho-sociale ains que du dispositif de formation desprofessionnels de santé. Cesconséquences, mal évaluées, sont indéniables même sill est prévisible que ces orientations seront régulées et nuancées par de multiples autres déterminants. Le travail de Chantal Kohler et coll. ${ }^{1}$ nous rappelle d'abord qu'en France, seuls les objectifs académiques à court terme sont explicitement pris en compte Conformément à une certaine conception républicaine du mérite, solidement établie, la sélection des futurs médecins françai sreposesur les résultats obtenus à un concours écrit anonyme. Diversaléas et vicissitudes, rapportés régulièrement à la une de la presse nationale la plus sérieuse $^{8,9,10,11}$, n'ont pas réussi à ébranler la foi des étudiants, de leurs parents et de leursfuturs malades en sa capacité à garantir à la fois une scrupuleuse équité entre les candidats et une forte probabilité de compétence des futurs professionnels sálectionnés $L a$ légitimité et l'efi cience sociales d'une telle procédure mériterai ent pourtant d'être questionnées

Ains, nous rappellet-on d'une part que, compte tenu de l'obligation de l'éudiant français de sins crire dans la (ou l'une des) faculté(s) de sa région académique, la réduction relative de la chance d'un étudiant de Dijon d'être admis en médeane est de 35 \% par rapport à celle d'un étudiant de Limoges Ains, d'autre part, l'analyse des résultats de l'étude nancéenne indiquet-elle que, sur les dix dernières années, près de $70 \%$ des étudiants admis à poursuivre leurs études de médecine ont dû redoubler leur première année, cette proportion ayant même tendanceà saccroître récemment pour atteindre près de $80 \%$ en 2000-2001. Ces étudiants redoublants et finalement admisn'étaient pourtant pas en situation d'échec académique ; les résultats concernant l'augmentation de la note moyenne d'une anné à l'autre montrent en effet que, pris globalement, presque tous avaient sans doute déjà obtenu une note supérieureà 10 sur 20 à l'issue de leur premier concours Les résultats du travail confirment encore que seulslesétudiantsissus de la série scientifiquedu baccalauréat d'études secondaires sont reçus au concours et que les mentions "très bien 》 ou «bien » obtenues à cet examen sont fortement pré dictives de leur réussite. II n'est certespasillogiquede chercher à identifier des étudiants qui aient une forte probabilité d'achever avec succès les longues études dans lesquelles ils souhaitent sengager. Une récente analyse systématique de la littérature ${ }^{12}$ montre que les résultats scolai resantérieursont, à cet égard, une bonne mais imparfaite valeur prédictive ; ils rendent compte d'environ $23 \%$ des diffé rences de performances académiques observées en premier et deuxième cycles (études « pré-graduées») maisseulement de $6 \%$ des résultatsobservésen troisième cycle (études " post-graduées »). II est, en tout cas, licite de sinterroger sur la valeur ajoutée du concours français de première année à l'égard d'un tel objectif ; la forte redondance de ses réultats avec ceux du baccalauréat d'études secondaires suggère, de fait, que les deux procédures identifient, peu ou prou, les mêmes caractéristiques individuelles. Audelà de la capacité à prédire les résultats acadé miques ultérieurs, une telle valeur ajoutée pourrait d'ailleurs être cherchée dans l'apti tude de la procé dure à identifier d'autres caractéristiques jugées importantes pour l'exercice futur de la profession médicale, telles que la capacité à résoudre un problème scientifique, à identifier un problème psychologique ou social, à formuler un dilemme éthique et à prendre, dans un contexte de conflit de valeurs, une décision judicieuse et réfléchie ${ }^{2}$. D'autres profes sions, telles que le corps des astronautes ou des cos- 
monaute ${ }^{13}$, en apparence fort éloi gnées de la méde cine mais qui ont auss à faire face à l'incertitude et à la complexité, ont développé des procédures d'évaluation objectives apparemment performantes d'un large panel de critères psychologiques, incluant les capacités d'adaptation et de flexibilité, de travail en équipe, de prise de décision en situation de stress ou encore de tolérance à l'isolement. En médecine, de telles procédures sont rarement misesen cuvreet certaines études ont montré l'échec du regard subjectif de psychiatres à prédire avec sécurité le profil de fonctionnement psychologique des futurs praticiens ${ }^{14}$.

A bien y réfléchir, il pourrait du reste être fallacieux d'exiger que l'étudiant démontre, dès le début de ses études, les qual ités attendues desa part lorsqu'il sera devenu un professionne à l'issue de la formation pour laquelle on le sálectionne; il y aurait mêmelà, dansune certaine mesure, un paradoxe pédagogique foncier sapparentant à un déni de ce que vise prédsément la dynamiqued'enseignement et d'apprentis sage Sauf à accepter de se positionner sur une conception obsolète plus ou moins fixiste et innéste de l'intelligence, il faut en effet considérer que les aptitudesne sont pas exclusivement desqualités pré existantes mais qu'eles sont auss des formes de compétences en développement, qu'elles sont modifiables à l'issue d'un apprentissage \& qu'elles doivent donc être enseignées ${ }^{15}$. II sagit ce faisant de reconnaître que, chez une personne, les facteurs génétiques et environnementaux sont susceptibles d'une interaction durable qui n'est pas limitée aux premières années de la vie U neautre conception erronéeserait par ailleurs de considérer que des stratégies et des compétences génériques, construites par hypothèse en dehors de contextes et de connaissances spécifiques -puisque préexistantes à la formation-, pourraient garantir le transfert ultérieur des apprentissages en stuation professionnelle authentique on sait en effet que les stratégies de résolution de problèmes ne peuvent sélaborer hors de contenus spécifiques mais que, par contre, de multiples situations particulières peuvent donner progressivement naissance, grâce à des interventions pédagogiques appropriées, à des aptitudes et à des compétences transférables ${ }^{16}$.
Les différentes règles adoptées respectivement, de par le monde, par les facultés de médecine lors des processus de sélection des éudiants, traduisent bien la variété des positionnements conceptuels possibles ${ }^{2}$. A une extrémité, certaines privilégient les mesures quantitatives et la démarche statistique à partir de tests standardisés et de scores de résultats acadé miques $A$ une autre extrémité, d'autres privilégient l'appréciation qualitative plus ou moins explicite, utilisent l'analyse au cas par cas et sautorisent des exceptions à la règle. En cohérence avec l'idée que beau coup d'incertitudes persistent, certaines facultés de médecine adoptent des règles de sélection en deux temps: elles vérifient d'abord, sur la base de résultats académiques, que les étudiants possèdent un «potentie » cognitif suffisant aprèsquoi le processus de sálection ne repose plus que sur une appréciation de traits personnes qualitatifs.

A l'issue d'une revue systématique de la littérature sur le suje, M cGaghie formule six recommandati ons concernant la sélection des étudiants en méde$\left.c_{n} e^{2}: 1\right)$ Les personnes responsables de la sálection dans les facultés de médecine devraient être spécifiquement formées à l'égard des enjeux, des problèmes et des perspectives de cette procédure ; 2) Les décisions de sélection devraient être faites sur la base d'informations vérifiables, objectives, relatives à des critères publicsqui soient l'expression d'unepol itique institutionnelle ; 3) Les dispositifs de sélection devraient être développés dans un esprit d'expérimentation plutôt que dans une logique de certitude, qui accepte que les procédures soient imparfaites mais qui cherche constamment à les améiorer ; 4) Les dispositifs de sélection devraient être adaptés aux contextes nationaux et les solutions retenues devraient avoir une forte pertinence écologique, tenant compte des expériences internationales sans pour autant les reproduire par simple conformisme; 5) Les procédures de sálection des étudiants devraient être développées en prenant en considération les contextes sociaux, politiques et économiques, compte tenu de leurs conséquences prévi siblesà court et à long terme ; 6) La sélection des éudiants en médecine devrait être conduite dans une perspective évolutive, prenant en compteà la foisles réultats de 
la recherche, les acquis des expérien ces développées et les changements intervenant dans le champ des valeurs sociales

En juin 2000, la France sest vue décerner une très enviable-et parfois contestée première placeau das sement de l'O rganisation mondiale de la santéselon la « performance globale du système de santé ». Eu égard à cetteposition, et comptetenu précisément des conséquences du choix des futurs médecins sur les caractéristiquesultérieures du système de santé, il et légitime de sinterroger quant à la pertinence d'un dispositif de sálection de ses étudiants en médecine qui n'a pratiquement pas changé depuis plus de 30 ans. La sélection des étudiants en médecine en France résulte d'un dispositif à certains égards bien réducteur quant à sesvaleurs implicites, qui privilé gie notamment la compétition, l'individualisme et le «talent académique »; ce dispositif n'est sans doute pas aussi équitable qu'il paraît puisque, malgré un renforcement constant du « verrouillage docimologique » qui connaîtra pourtant -à n'en pas douter- d'autres bavures, il est marqué par desirré gularités récurrentes et induit, par exemple, des inégalités régionales; ce di spositif est enfin générateur d'un immense gâchis éducatif en contraignant à redoubler, alors qu'ils ne sont pas en situation d'échec, en moyenne 70 pour cent des éudiants de chacune des promotions sélectionnées Les citoyens seraient fondés à demander des comptes à l'égard d'un tel constat. Les enseignants des facultés de médecine, pour ce qui lesconcerne, nepeuvent assurément pas śaffranchir d'une réflexion approfondie en la matière. Le projet annoncé d'une formation initiale partiellement conjointe des différents profes sionnels de santé pourrait être prochainement une occasion favorable à saisir pour initier un tel débat.

Jean JO U Q UAN

mailto:jean.jouquan@chu-brest.fr
1. Kholer C, Braun M, Mari G, Roland J. Evolution du profil des étudiants ayant passé le concours de PCEM 1 à la faculté de médecine de $\mathrm{N}$ ancy de 1992 à 2001. Pédagogie M édicale $2003 ; 4: 12-17$.

2. M CGaghie WC. Student selection. In : G. Norman, CPM van der Vleuten, DI N ewble (Eds). International $\mathrm{H}$ andbook of Research in Medical Education. Kluwer Academic Publishers : Dordrecht, The Netherlands : $2002 ; 303-336$.

3. Deng Yizhong. D evelopment of medical education in China. Acad Med 1990 ; 65 : 512514.

4. Drabkin IE. On medical education in Greece and Rome. Bull H ist Med 1944 ; 15 : 333351.

5. Powis DA. Selecting medical students. Med Educ $1994 ; 28: 443-469$.

6. M CM anus IC. Factors affecting likehood of applicants being offered a place in medical schools in the United Kingdom in 1996 and 1997 : retrospective study. BMJ 1998 ; 317 : 1111-1117.

7. Davidson RC, Lewis EL. Affirmative action and other special consideration admissions at the University of California, D avis, School of M edicine. JAM A 1997 ; 278 : 1153-1158.
8. D elberghe $M$. Le concours de première année de médecine est à nouveau mis en cause. Le M onde, 13 juin 1997.

9. Incidents survenus lors des épreuves du concours de fin de première année des études médicales. Le M onde, 26 octobre 1999.

10. O rtscheidt R. A près l'annulation du concours de médecine. Montpellier: Kafka à la fac. Libération, 23 octobre 1999.

11. Allain P-H. Brest : le concours de médecine recalé. Sept mois après, une des épreuves de première année doit être réorganisée. Libération, 10 mars 2000.

12. Ferguson $E$, James $D, M$ adeley $L$. Factors associated with success in medical school : systematic review of the literature. BMJ 2002 ; $324: 952-957$.

13. Santy PA. Choosing the right stuff : The psychological selection of astronauts and cosmonauts. 1996, Westport, CT : Praeger.

14. Aldrich CK. The clouded crystal ball : A 35year follow-up of psychiatrists'predictions. Am J Psychiatry $1986 ; 143$ : 45-49.

15. Sternberg RJ . A bilities are forms of developing expertise. Educational Researcher $1998 ; 27$ : 11-20.

16. Tardif J. Le transfert des apprentissages. 1999, M ontréal, PQ : Les Editions Logiques. 